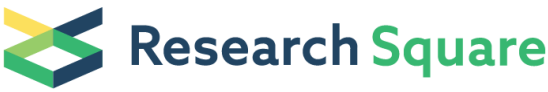 \\ Preprints are preliminary reports that have not undergone peer review. \\ They should not be considered conclusive, used to inform clinical practice, or referenced by the media as validated information.
}

\section{Association Between Clinical Risk Factors and Coronary Artery Lesions in Children with Kawasaki Disease}

\section{Xiangna Yang}

Pediatrics of Traditional Chinese Medicine, The first clinical medical college of Guangzhou University of Chinese medicine囚Guangzhou 510405, Guangdong

\section{Xiaogang Chen ( $\square$ wyzfz@163.com )}

Department of Pediatrics, First Affiliated Hospital of Guangzhou University of Traditional Chinese Medicine, Guangzhou 510405, Guangdong

\section{Jian Deng}

Department of Pediatrics of Traditional Chinese Medicine, Guangzhou Women and Children Medical Center, Guangzhou 510623, Guangdong

\section{Yanfei Wang}

Department of Cardiology, Guangzhou Women and Children Medical Center, Guangzhou 510623, Guangdong

Ziyi Li

Center for Clinical Epidemiology and Methodology, Guangdong Second Provincial General Hospital, Guangzhou, China

\section{Research Article}

Keywords: Kawasaki disease, coronary artery lesions, pediatric, biomarker

Posted Date: February 4th, 2022

DOl: https://doi.org/10.21203/rs.3.rs-1212215/v1

License: (a) (i) This work is licensed under a Creative Commons Attribution 4.0 International License. Read Full License 


\section{Abstract}

Background: Kawasaki disease (KD) is one of the leading causes of acquired coronary artery diseases in childhood. In KD patients, the evidence on the association between easy-to-assess clinical risk factors and development of coronary artery lesion (CAL). Our study aimed to determine the clinical biomarkers that were associated with latent occurrence of CAL in pediatric patients with $\mathrm{KD}$.

Methods: 260 pediatric KD patients were recruited. The outcome of our study is CAL, including coronary dilation, coronary aneurysm, and intimal thickening of coronary arteries. Data of general information, medical history, results of laboratory examinations and echocardiography data were collected. Logistic regression method was carried out to explore the associations between biomarkers and each CAL.

Results: Sixty-six (25.4\%), thirty-nine (15\%) and fifty-six patients $(21.5 \%)$ of the participants had coronary dilation, coronary aneurysm, and intimal thickening of coronary arteries, respectively. Single-variable analysis found age, gender, duration of fever, time of initial use of IVIG, ESR, WBC, time of platelet increase, maximum value of platelet, albumin and IgG level were associated with CAL. In multivariable logistic analysis, younger in age and male were associated with all three outcomes of CAL. Lower serum albumin level and later initial use of IVIG were linked to higher risk of coronary dilation and coronary aneurysm.

Conclusion: In a Chinese pediatric population of KD, younger in age, male, lower serum albumin lever, and later initial use of IVIG were risk factors of CAL. Our findings had high practicability to be implemented in clinical practice, however, is to be verified by future studies.

\section{Background}

Kawasaki disease (KD), also called mucocutaneous lymph node syndrome, is an acute, febrile vasculitis disorder which is one of the leading causes of acquired coronary artery diseases in childhood, especially in pediatric patients younger than five years.

The epidemiology of KD varies greatly by geographic location. In western countries, the annual incidence of KD for children under five years old ranged from 4 to 25 per 100000 [1-3]. Nevertheless, the incidence of KD was higher among north and east Asians. For example, in Japan, the accordingly incidence in children under 5 was 243.1 per 100 000 in 2011, and 264.8 per 100000 in 2012 [4]. In China, the annual incidence ranged from 5 to 69 per 100000 [5-8]. Meanwhile, the incidence in Asian area was on increase [8-10]. Besides regional difference, the higher incidence in Japanese children was also linked to the ethnic variation. It was reported that among all ethnic groups in children under 5 years old in Hawaii, children of Japanese descent had the highest incidence (210.5 per 100 000), while Caucasian children had the lowest (13.7 per 100 000) [11]. The above-mentioned evidence highlighted the importance of relevant studies in Asian countries.

$\mathrm{KD}$ is one of the most common causes of acquired heart disease in children worldwide [9], furthermore, it is a condition with potential life-long health affect [12]. The long-term prognosis is determined by the level of coronary artery lesion (CAL) [13]. For instance, Japanese studies reported that the standardized mortality ratios in KD patients with cardiac sequelae, for example coronary artery aneurysms, were higher than those without [14, 15]. Moreover, in KD patients with coronary artery aneurysms and stenoses, sudden death of myocardial infarction (MI) can occur many years later in childhood and even in adulthood [16]. Therefore, for the purpose of giving effective and targeted treatment which could possibly have life-long benefits, it is important to recognize patients who are at elevated risk with CAL in the early stage of KD. Determine easy-to-assess clinical characteristics and biomarkers, which were 
associated with the progression of CAL, could help pediatrician provide timely preventive and therapeutic measures to reduce the prevalence of CAL. Clinical characteristics and biomarkers such as laboratory findings are useful in supporting a diagnosis of KD [17], however, the evidence on the association between them and development of CAL in pediatric KD patients are lacking.

Given the scarce findings and the clinical importance on clinical risk factors CAL in KD patients, we conducted the current study, aiming to determine the clinical biomarkers that were associated with latent occurrence of CAL in pediatric patients with KD. Multiple outcomes (coronary dilation, coronary aneurysm, and intimal thickening of coronary arteries) were involved in our study, to provide more comprehensive evidence on the topic.

\section{Methods}

\section{Participants}

We investigated pediatric patients with complete KD who were admitted at the Department of Cardiology in Guangzhou Women's and Children's Medical Center from June to December 2017. The inclusion criteria for our study were: (1) patients that were diagnosed as complete KD for the first time, (2) patients that had complete data on general information, biomarkers, and outcome factors, and (3) the legal guardian of the patient gave informed consent to take part in the study. Patients did not have insufficient evidence to be diagnosed as KD, with incomplete $K D$, in recurrent episode of KD, and those did not have complete data were excluded from our study. Based on these inclusion criteria, a total of 260 subjects were included. According to Diagnosis, treatment, and long-term management of Kawasaki disease: a statement for health professionals from the Committee on Rheumatic Fever, Endocarditis and Kawasaki Disease, Council on Cardiovascular Disease in the Young by American Heart Association [18], our diagnostic criteria for complete KD was based on 5 principal clinical features, namely conjunctival injection, rash, strawberry tongue or red lips, neck lymphadenopathy, and edema and erythema of the hand and foot. Children were diagnosed as complete KD if they had 4 or 5 of these aforementioned symptoms, with fever that lasted for 5 or more days. Incomplete KD were diagnosed as 2 or there of the symptoms with fever for 5 or more days.

\section{Outcome}

The outcome of our study is CAL, including coronary dilation, coronary aneurysm, and intimal thickening of coronary arteries. According to Diagnosis, Treatment, and Long-Term Management of Kawasaki Disease: A Scientific Statement for Health Professionals From the American Heart Association (2017) [19], the coronary dilation and coronary aneurysm were diagnosed and classified based on Z values: (1) No involvement: always <2, (2) Dilation only: 2 to $<2.5$; or if initially $<2$, a decrease in Z score during follow-up $\geq 1$, (3) Small aneurysm: $\geq 2.5$ to $<5$, (4) Medium aneurysm: $\geq 5$ to $<10$, and absolute dimension $<8 \mathrm{~mm}$, and (5) Large or giant aneurysm: $\geq 10$, or absolute dimension $\geq 8 \mathrm{~mm}$. Intimal thickening of coronary arteries were assessed with optical coherence tomography. Data on incidence, severity and location of each CAL were collected and included into the analysis.

\section{Data collection}

Data of the recruited patients were extracted from medical records of Hospital Information System, which included the patient's general information, history of present illness, results of laboratory examinations, echocardiography data and history of medication usage. The laboratory examinations were measured upon admission. Intravenous immunoglobulin (IVIG) resistance was defined as a body temperature of $\geq 38.0^{\circ} \mathrm{C}$ that lasted between 36 hours and 7 days, or recurrence of fever after the completion of IVIG infusion [19].

\section{Ethics}


The protocol of the present study was reviewed and approved by the Guangzhou Women and Children Medical Center Ethics Committee (2021241A01) and was conducted according to the International Ethical Guidelines for Research Involving Human Subjects stated in the Declaration of Helsinki. The children's families provide written informed consent.

\section{Statistical analysis}

Data were described as the mean and standard deviation (SD) for continuous variables, and number and percentages for categorical variables. Continuous variables were compared using t-tests to estimate the significance of differences between patients with and without certain CAL. Categorical variables were compared using $\chi 2$ tests. Potential predictors were selected for each CAL separately. Variables presented statistically significance for at least one outcome were included in the multivariable logistic regression models. Logistic regression was carried out to explore the associations between potential risk factors and each CAL. Multivariable logistic models were established to identify the risk factors. A sensitivity analysis was carried out by adding the variable "whether had IVIG resistance" into the final models, to explore whether IVIG resistance had modification effect of the relationships that were found in the main analysis. Statistical analyses were performed using STATA software, $p$ values that $<0.05$ were considered statistically significant.

\section{Results}

\section{General information of the participants}

In total, 260 patients with infantile KD were recruited in our study, with a mean age of 24.05 month (SD = 21.85 month), and a ratio of gender (male: female) as 172:88. The mean duration of fever was 7.52 days (SD = 3.27 days). The mean time of initial use of IVIG was 6.44 days (SD = 2.12 days) after the onset of KD. Eighty-four percent of the participants used IVIG 1 time $(n=218)$, and $3(1.2 \%)$ of the total sample never used IVIG. Twenty-four children had IVIG resistance, accounted for $9.2 \%$ of the total participants. The detailed general information is displayed in Table 1 . 
Table 1

Description of general characteristics

\begin{tabular}{|ll|}
\hline Characteristics & Mean (SD)/n (\%) \\
\hline Age (month) & $24.05(21.85)$ \\
\hline Gender & $172(66.2 \%)$ \\
\hline Male & $88(33.9 \%)$ \\
\hline Blood type & \\
\hline A & $76(29.2 \%)$ \\
\hline B & $67(25.8 \%)$ \\
\hline O & $108(41.5 \%)$ \\
\hline AB & $9(3.5 \%)$ \\
\hline Duration of fever (day) & $7.52(3.27)$ \\
\hline Time of initial use of IVIG & $6.44(2.12)$ \\
\hline Frequency of IVIG usage (time) & $1.15(0.43)$ \\
\hline 0 & $3(1.2 \%)$ \\
\hline 1 & $218(83.9 \%)$ \\
\hline 2 & $35(13.5 \%)$ \\
\hline 3 & $4(1.5 \%)$ \\
\hline IVIG resistance & $24(9.23 \%)$ \\
\hline IVIG: intravenous immunoglobulin; SD: standard deviation \\
\hline
\end{tabular}

\section{Description of coronary artery lesion}

Prevalence of CALs is displayed in Table 2. Sixty-six (25.4\%) of the participants had coronary dilation, with a mean time of first occurrence of 34.31 days (SD = 70.34 days) after the onset of KD. In $34(53.1 \%)$ patients, coronary dilation located only in left coronary arteries. Thirty-nine (15\%) of the participants suffered from coronary aneurysm, with a mean time of first occurrence of 11.73 days (SD = 6.34 days) after the onset of KD. In $22(56.4 \%)$ patients, the coronary aneurysm located in both left and right coronary arteries. Most of the patients had coronary aneurysm of small size (84.6\%), and 4/1/1 patients that had medium/large/extra-large coronary aneurysm, respectively. The mean time of regression of coronary aneurysm was 50.13 days (SD $=63.63$ days) after the onset of KD. Fifty-six patients (21.5\%) had intimal thickening of coronary arteries. Most participants had intimal thickening only in left coronary arteries, which is similar to coronary dilation. One patient had thrombus. The mean time of total recovery was 81.27 days $(S D=261$ days $)$. 
Table 2

Description of coronary artery lesion

\begin{tabular}{|c|c|}
\hline Status of coronary artery lesion & Mean (SD)/n (\%) \\
\hline Coronary aneurysm & $39(15 \%)$ \\
\hline Time of occurrence of coronary aneurysm (day after the onsite of KD) & $11.71(6.34)$ \\
\hline \multicolumn{2}{|l|}{ Size of coronary aneurysm } \\
\hline Small & $33(84.6 \%)$ \\
\hline Medium & $4(10.3 \%)$ \\
\hline Large & $1(2.6 \%)$ \\
\hline Extra large & $1(2.6 \%)$ \\
\hline \multicolumn{2}{|l|}{ Location of coronary aneurysm } \\
\hline Left only & $12(30.8 \%)$ \\
\hline Right only & $5(12.8 \%)$ \\
\hline Left and right & $22(56.4 \%)$ \\
\hline Coronary aneurysm regression time (day after the onsite of KD) & $50.13(63.63)$ \\
\hline Thrombus & $1(0.4 \%)$ \\
\hline Coronary dilation & $66(25.4 \%)$ \\
\hline Time of occurrence of coronary dilation (day after the onsite of KD) & $34.31(70.34)$ \\
\hline \multicolumn{2}{|l|}{ Location of coronary dilation } \\
\hline Left only & $34(53.1 \%)$ \\
\hline Right only & $5(7.8 \%)$ \\
\hline Left and right & $25(39.1 \%)$ \\
\hline Intimal thickening & $56(21.5 \%)$ \\
\hline \multicolumn{2}{|l|}{ Location of coronary intimal thickening } \\
\hline Left only & $27(48.2 \%)$ \\
\hline Right only & $7(12.5 \%)$ \\
\hline Left and right & $22(39.3 \%)$ \\
\hline Time of recovery (day after the onsite of KD) & $81.27(261.95)$ \\
\hline
\end{tabular}

\section{Comparison of clinical biomarkers between participants with and without CAL}

Duration of fever, and time of initial usage of IGIV were positively corelated with the incidence of all the three CALs, while month of age and serum albumin level were negatively corelated. Gender was associated only with coronary dilation, the percentage of boys in those with coronary dilation was higher than without $(78.8 \%$ v.s. $61.9 \%, p=0.012)$. 
Erythrocyte sedimentation rate (ESR) was positively linked to only coronary aneurysm. White blood cell count (WBC) was positively related to coronary aneurysm and intimal thickening. Time of the increase in platelets was negatively correlated with coronary dilation, and the maximum value of platelets was positively related to coronary dilation and coronary aneurysm. IgG level was only positively related to intimal thickening. Results of the comparison of clinical biomarkers between participants with and without CAL are displayed in Table 3. 
Table 3

Comparison of clinical biomarkers between participants with and without CAL

\begin{tabular}{|c|c|c|c|c|c|c|c|c|c|}
\hline \multirow{2}{*}{$\begin{array}{l}\text { Potential } \\
\text { Risk } \\
\text { Factors }\end{array}$} & \multicolumn{2}{|c|}{ Mean (SD)/n (\%) } & \multirow[t]{2}{*}{$p$ value } & \multicolumn{2}{|c|}{ Mean (SD)/n (\%) } & \multirow[t]{2}{*}{$p$ value } & \multicolumn{2}{|c|}{ Mean (SD)/n (\%) } & \multirow{2}{*}{$\mathrm{p}_{\text {value }}$} \\
\hline & CD & Non-CD & & $\mathrm{CA}$ & Non-CA & & $\mathrm{CIT}$ & Non-CIT & \\
\hline Age & $\begin{array}{l}17.5 \\
(16.63)\end{array}$ & $\begin{array}{l}26.27 \\
(22.98)\end{array}$ & $0.005^{\star}$ & $\begin{array}{l}14.82 \\
(15.99)\end{array}$ & $\begin{array}{l}25.67 \\
(22.37)\end{array}$ & $0.004^{*}$ & $\begin{array}{l}15.93 \\
(14.61)\end{array}$ & $\begin{array}{l}26.27 \\
(22.98)\end{array}$ & $0.002 *$ \\
\hline \multicolumn{10}{|l|}{ Gender } \\
\hline Male & $\begin{array}{l}52 \\
(78.8 \%)\end{array}$ & $\begin{array}{l}120 \\
(61.9 \%)\end{array}$ & $0.012^{\star}$ & $\begin{array}{l}30 \\
(76.9 \%)\end{array}$ & $\begin{array}{l}142 \\
(64.3 \%)\end{array}$ & 0.123 & $\begin{array}{l}43 \\
(76.8 \%)\end{array}$ & $\begin{array}{l}129 \\
(63.2 \%)\end{array}$ & 0.058 \\
\hline Female & $\begin{array}{l}14 \\
(21.2 \%)\end{array}$ & $\begin{array}{l}74 \\
(38.1 \%)\end{array}$ & & $\begin{array}{l}9 \\
(23.1 \%)\end{array}$ & $\begin{array}{l}79 \\
(35.7 \%)\end{array}$ & & $\begin{array}{l}13 \\
(23.2 \%)\end{array}$ & $\begin{array}{l}75 \\
(36.8 \%)\end{array}$ & \\
\hline \multicolumn{10}{|l|}{$\begin{array}{l}\text { Blood } \\
\text { type }\end{array}$} \\
\hline A & $\begin{array}{l}18 \\
(27.3 \%)\end{array}$ & $\begin{array}{l}58 \\
(29.9 \%)\end{array}$ & 0.968 & $\begin{array}{l}9 \\
(23.1 \%)\end{array}$ & $\begin{array}{l}67 \\
(30.3 \%)\end{array}$ & 0.703 & $\begin{array}{l}12 \\
(21.4 \%)\end{array}$ & $\begin{array}{l}64 \\
(31.4 \%)\end{array}$ & 0.450 \\
\hline B & $\begin{array}{l}18 \\
(27.3 \%)\end{array}$ & $\begin{array}{l}49 \\
(25.3 \%)\end{array}$ & & $\begin{array}{l}12 \\
(30.8 \%)\end{array}$ & $\begin{array}{l}55 \\
(24.9 \%)\end{array}$ & & $\begin{array}{l}18 \\
(32.1 \%)\end{array}$ & $\begin{array}{l}49 \\
(24 \%)\end{array}$ & \\
\hline 0 & $\begin{array}{l}28 \\
(42.4 \%)\end{array}$ & $\begin{array}{l}80 \\
(41.2 \%)\end{array}$ & & $\begin{array}{l}16 \\
(41 \%)\end{array}$ & $\begin{array}{l}92 \\
(41.6 \%)\end{array}$ & & $\begin{array}{l}24 \\
(42.9 \%)\end{array}$ & $\begin{array}{l}84 \\
(41.2 \%)\end{array}$ & \\
\hline$A B$ & $2(3 \%)$ & 7 (3.6\%) & & $2(5.1 \%)$ & 7 (3.2\%) & & $2(3.6 \%)$ & 7 (3.4\%) & \\
\hline $\begin{array}{l}\text { Duration } \\
\text { of fever } \\
\text { (day) }\end{array}$ & $\begin{array}{l}8.53 \\
(3.8)\end{array}$ & $\begin{array}{l}7.18 \\
(2.99)\end{array}$ & $0.004^{\star}$ & $\begin{array}{l}9.56 \\
(4.84)\end{array}$ & $\begin{array}{l}7.16 \\
(2.76)\end{array}$ & $<0.001^{*}$ & $\begin{array}{l}8.8 \\
(3.98)\end{array}$ & $\begin{array}{l}7.17 \\
(2.95)\end{array}$ & $0.001^{*}$ \\
\hline $\begin{array}{l}\text { Time of } \\
\text { initial use } \\
\text { of IVIG } \\
\text { (day after } \\
\text { the onsite } \\
\text { of KD) }\end{array}$ & $\begin{array}{l}7.22 \\
(2.55)\end{array}$ & $\begin{array}{l}6.17 \\
(1.89)\end{array}$ & $0.001^{*}$ & $\begin{array}{l}7.77 \\
(3.44)\end{array}$ & $\begin{array}{l}6.2 \\
(1.69)\end{array}$ & $<0.001^{\star}$ & $\begin{array}{l}7.22 \\
(2.51)\end{array}$ & $\begin{array}{l}6.22 \\
(1.96)\end{array}$ & $0.002 *$ \\
\hline $\begin{array}{l}\text { ESR } \\
(\mathrm{mm} / \mathrm{h})\end{array}$ & $\begin{array}{l}38.26 \\
(24.92)\end{array}$ & $\begin{array}{l}36.77 \\
(19.72)\end{array}$ & 0.622 & $\begin{array}{l}43.36 \\
(25.32)\end{array}$ & $\begin{array}{l}36.05 \\
(20.16)\end{array}$ & $0.046^{\star}$ & $\begin{array}{l}37.96 \\
(24.79)\end{array}$ & $\begin{array}{l}36.92 \\
(20.06)\end{array}$ & 0.745 \\
\hline $\begin{array}{l}\text { hsCRP } \\
\text { (mg/L) }\end{array}$ & $\begin{array}{l}119.96 \\
(167.4)\end{array}$ & $\begin{array}{l}95.13 \\
(91.94)\end{array}$ & 0.133 & $\begin{array}{l}134.76 \\
(157.57)\end{array}$ & $\begin{array}{l}95.55 \\
(106.36)\end{array}$ & 0.051 & $\begin{array}{l}93.81 \\
(65.95)\end{array}$ & $\begin{array}{l}103.52 \\
(126.33)\end{array}$ & 0.580 \\
\hline $\begin{array}{l}\text { WBC } \\
\text { count } \\
\left(\times 10^{9} / L\right)\end{array}$ & $\begin{array}{l}18.49 \\
(6.5)\end{array}$ & $\begin{array}{l}16.98 \\
(5.5)\end{array}$ & 0.067 & $\begin{array}{l}20.9 \\
(6.21)\end{array}$ & $\begin{array}{l}16.74 \\
(5.5)\end{array}$ & $<0.001^{\star}$ & $\begin{array}{l}18.86 \\
(6.61)\end{array}$ & $\begin{array}{l}16.95 \\
(5.5)\end{array}$ & $0.029 *$ \\
\hline $\mathrm{HB}(\mathrm{g} / \mathrm{L})$ & $\begin{array}{l}104.15 \\
(112.47)\end{array}$ & $\begin{array}{l}98.43 \\
(11)\end{array}$ & 0.484 & $\begin{array}{l}87.54 \\
(11.64)\end{array}$ & $\begin{array}{l}102.06 \\
(61.61)\end{array}$ & 0.144 & $\begin{array}{l}90.8 \\
(11.72)\end{array}$ & $\begin{array}{l}102.38 \\
(64.09)\end{array}$ & 0.180 \\
\hline
\end{tabular}

${ }^{\star} p<0.05$

ALT: alanine aminotransferase; CA: Coronary aneurysm; CD: Coronary dilation; CIT: Coronary intimal thickening; CK-MB: creatine kinase isoenzyme; DBil: direct bilirubin; ESR: erythrocyte sedimentation rate; HB: hemoglobin; hsCRP: hypersensitive C-reactive protein; IBil: indirect bilirubin; IVIG: intravenous immunoglobulin; LDH: lactic dehydrogenase; PLT: platelet; SD: Standard deviation; TBA: total bile acid; TBil: total bilirubin; WBC: white blood cell count 


\begin{tabular}{|c|c|c|c|c|c|c|c|c|c|}
\hline \multirow{2}{*}{$\begin{array}{l}\text { Potential } \\
\text { Risk } \\
\text { Factors }\end{array}$} & \multicolumn{2}{|c|}{ Mean (SD)/n (\%) } & \multirow[t]{2}{*}{$p$ value } & \multicolumn{2}{|c|}{ Mean (SD)/n (\%) } & \multirow[t]{2}{*}{$p$ value } & \multicolumn{2}{|c|}{ Mean (SD)/n (\%) } & \multirow{2}{*}{$\begin{array}{l}p \\
\text { value }\end{array}$} \\
\hline & $C D$ & Non-CD & & $\mathrm{CA}$ & Non-CA & & CIT & Non-ClT & \\
\hline $\begin{array}{l}\text { Time of } \\
\text { PLT } \\
\text { increase } \\
\text { (day after } \\
\text { the onsite } \\
\text { of KD) }\end{array}$ & $\begin{array}{l}83.27 \\
(264.2)\end{array}$ & $\begin{array}{l}207.02 \\
(398.3)\end{array}$ & $0.019 *$ & $\begin{array}{l}85.28 \\
(267.23)\end{array}$ & $\begin{array}{l}191.55 \\
(386.25)\end{array}$ & $0.100 *$ & $\begin{array}{l}114.46 \\
(309.2)\end{array}$ & $\begin{array}{l}192.39 \\
(386.88)\end{array}$ & 0.166 \\
\hline $\begin{array}{l}\text { Maximum } \\
\text { PLT }\end{array}$ & $\begin{array}{l}679.41 \\
(190.08)\end{array}$ & $\begin{array}{l}580.72 \\
(193.41)\end{array}$ & $<0.001^{\star}$ & $\begin{array}{l}709.85 \\
(198.81)\end{array}$ & $\begin{array}{l}587.4 \\
(191.28)\end{array}$ & $<0.001^{\star}$ & $\begin{array}{l}650.95 \\
(192.75)\end{array}$ & $\begin{array}{l}593.37 \\
(196.76)\end{array}$ & 0.053 \\
\hline $\begin{array}{l}\text { Albumin } \\
\text { (g/L) }\end{array}$ & $\begin{array}{l}34.93 \\
(4.66)\end{array}$ & $\begin{array}{l}36.68 \\
(4.36)\end{array}$ & $0.006^{*}$ & $\begin{array}{l}33.95 \\
(5.03)\end{array}$ & $\begin{array}{l}36.65 \\
(4.28)\end{array}$ & $<0.001^{*}$ & $\begin{array}{l}35.03 \\
(4.78)\end{array}$ & $\begin{array}{l}36.57 \\
(4.37)\end{array}$ & $0.024 *$ \\
\hline ALT (U/L) & $\begin{array}{l}74.03 \\
(133.05)\end{array}$ & $\begin{array}{l}68.16 \\
(142.15)\end{array}$ & 0.769 & $\begin{array}{l}67.3 \\
(74.92)\end{array}$ & $\begin{array}{l}70.06 \\
(148.3)\end{array}$ & 0.909 & $\begin{array}{l}65.76 \\
(79.76)\end{array}$ & $\begin{array}{l}70.72 \\
(152.18)\end{array}$ & 0.815 \\
\hline $\begin{array}{l}\text { TBil } \\
(\mu \mathrm{mol} / \mathrm{L})\end{array}$ & $\begin{array}{l}7.63 \\
(12.34)\end{array}$ & $\begin{array}{l}8.19 \\
(12.28)\end{array}$ & 0.751 & $\begin{array}{l}7.54 \\
(14.06)\end{array}$ & $\begin{array}{l}8.14 \\
(11.96)\end{array}$ & 0.779 & $\begin{array}{l}7.34 \\
(12.05)\end{array}$ & $\begin{array}{l}8.24 \\
(12.36)\end{array}$ & 0.630 \\
\hline $\begin{array}{l}\text { DBil } \\
(\mu \mathrm{mol} / \mathrm{L})\end{array}$ & $\begin{array}{l}4.55 \\
(10.3)\end{array}$ & $\begin{array}{l}4.77 \\
(10.48)\end{array}$ & 0.886 & $\begin{array}{l}4.6 \\
(12.04)\end{array}$ & $\begin{array}{l}4.73 \\
(10.13)\end{array}$ & 0.943 & $\begin{array}{l}4.31 \\
(10.23)\end{array}$ & $\begin{array}{l}4.82 \\
(10.48)\end{array}$ & 0.744 \\
\hline $\begin{array}{l}\text { IBil } \\
(\mu \mathrm{mol} / \mathrm{L})\end{array}$ & $\begin{array}{l}6.31 \\
(26)\end{array}$ & $\begin{array}{l}3.41 \\
(2.64)\end{array}$ & 0.128 & $\begin{array}{l}2.94 \\
(2.24)\end{array}$ & $\begin{array}{l}4.35 \\
(14.33)\end{array}$ & 0.539 & $\begin{array}{l}3.03 \\
(2.26)\end{array}$ & $\begin{array}{l}4.44 \\
(14.87)\end{array}$ & 0.486 \\
\hline $\begin{array}{l}\text { TBA } \\
(\mu \mathrm{mol} / \mathrm{L})\end{array}$ & $\begin{array}{l}13.69 \\
(37.28)\end{array}$ & $\begin{array}{l}16.79 \\
(33.63)\end{array}$ & 0.540 & $\begin{array}{l}11.92 \\
(15.59)\end{array}$ & $\begin{array}{l}16.78 \\
(36.87)\end{array}$ & 0.419 & $\begin{array}{l}10.29 \\
(14.22)\end{array}$ & $\begin{array}{l}17.54 \\
(37.96)\end{array}$ & 0.174 \\
\hline $\lg G(g / L)$ & $\begin{array}{l}9.58 \\
(8.03)\end{array}$ & $\begin{array}{l}8.14 \\
(5.48)\end{array}$ & 0.105 & $\begin{array}{l}9.43 \\
(8.46)\end{array}$ & $\begin{array}{l}8.34 \\
(5.78)\end{array}$ & 0.317 & $\begin{array}{l}10.16 \\
(9.87)\end{array}$ & $\begin{array}{l}8.05 \\
(4.73)\end{array}$ & $0.025^{\star}$ \\
\hline $\lg A(g / L)$ & $\begin{array}{l}0.74 \\
(0.57)\end{array}$ & $\begin{array}{l}0.72 \\
(0.58)\end{array}$ & 0.855 & $\begin{array}{l}0.74 \\
(0.64)\end{array}$ & $\begin{array}{l}0.72 \\
(0.56)\end{array}$ & 0.839 & $\begin{array}{l}0.66 \\
(0.45)\end{array}$ & $\begin{array}{l}0.74 \\
(0.61)\end{array}$ & 0.310 \\
\hline $\operatorname{lgM}(g / L)$ & $\begin{array}{l}1.06 \\
(0.54)\end{array}$ & $\begin{array}{l}1.16 \\
(0.53)\end{array}$ & 0.213 & $1(0.57)$ & $\begin{array}{l}1.16 \\
(0.53)\end{array}$ & 0.095 & $\begin{array}{l}1.05 \\
(0.59)\end{array}$ & $\begin{array}{l}1.16 \\
(0.52)\end{array}$ & 0.188 \\
\hline $\begin{array}{l}\lg E \\
(\mathrm{IU} / \mathrm{mL})\end{array}$ & $\begin{array}{l}100.06 \\
(172.21)\end{array}$ & $\begin{array}{l}174.79 \\
(371.48)\end{array}$ & 0.116 & $\begin{array}{l}85.69 \\
(126.48)\end{array}$ & $\begin{array}{l}168.2 \\
(356.77)\end{array}$ & 0.155 & $\begin{array}{l}87.5 \\
(133.82)\end{array}$ & $\begin{array}{l}174.58 \\
(368.18)\end{array}$ & 0.084 \\
\hline LDH (U/L) & $\begin{array}{l}275.62 \\
(94.07)\end{array}$ & $\begin{array}{l}273.71 \\
(82.87)\end{array}$ & 0.876 & $\begin{array}{l}253 \\
(44.62)\end{array}$ & $\begin{array}{l}277.84 \\
(90.46)\end{array}$ & 0.099 & $\begin{array}{l}273.07 \\
(105.11)\end{array}$ & $\begin{array}{l}274.5 \\
(79.94)\end{array}$ & 0.913 \\
\hline $\begin{array}{l}\text { CK-MB } \\
\text { (U/L) }\end{array}$ & $\begin{array}{l}19.26 \\
(12.09)\end{array}$ & $\begin{array}{l}23.02 \\
(32.72)\end{array}$ & 0.362 & $\begin{array}{l}17.84 \\
(6.82)\end{array}$ & $\begin{array}{l}22.79 \\
(31.13)\end{array}$ & 0.331 & $\begin{array}{l}23.55 \\
(33.72)\end{array}$ & $\begin{array}{l}21.67 \\
(27.56)\end{array}$ & 0.670 \\
\hline \multicolumn{10}{|l|}{${ }^{\star} p<0.05$} \\
\hline \multicolumn{10}{|c|}{$\begin{array}{l}\text { ALT: alanine aminotransferase; CA: Coronary aneurysm; CD: Coronary dilation; CIT: Coronary intimal thickening; } \\
\text { CK-MB: creatine kinase isoenzyme; DBil: direct bilirubin; ESR: erythrocyte sedimentation rate; HB: hemoglobin; } \\
\text { hsCRP: hypersensitive C-reactive protein; IBil: indirect bilirubin; IVIG: intravenous immunoglobulin; LDH: lactic } \\
\text { dehydrogenase; PLT: platelet; SD: Standard deviation; TBA: total bile acid; TBil: total bilirubin; WBC: white blood } \\
\text { cell count }\end{array}$} \\
\hline
\end{tabular}

\section{Multivariable analyses}

In the main analysis (Table 4), age $(\mathrm{OR}=0.975,95 \% \mathrm{Cl}: 0.955$ to $0.994, \mathrm{P}$ value $=0.012)$, being female $(\mathrm{OR}=0.304$, $95 \% \mathrm{Cl}: 0.142$ to $0.648, \mathrm{P}$ value $=0.002)$ and serum albumin level $(\mathrm{OR}=0.926,95 \% \mathrm{Cl}: 0.858$ to $0.998, \mathrm{p}$ value $=0.045)$ were found to be negatively linked with the risk of coronary dilation, while time of initial use of IVIG $(O R=1.255,95 \%$ 
$\mathrm{Cl}: 1.047$ to $1.503, \mathrm{p}$ value $=0.014)$ was positively linked. Age $(\mathrm{OR}=0.962,95 \% \mathrm{Cl}: 0.933$ to $0.991, \mathrm{P}$ value $=0.012)$ and serum albumin level $(\mathrm{OR}=0.890,95 \% \mathrm{Cl}: 0.809$ to $0.979, \mathrm{p}$ value $=0.017)$ were found to be negatively linked with the risk of coronary aneurysm, while WBC $(\mathrm{OR}=1.088,95 \% \mathrm{Cl}: 1.012$ to $1.169, \mathrm{p}$ value $=0.023)$ and time of initial use of IVIG (OR $=1.214,95 \% \mathrm{Cl}: 1.003$ to 1.469 , p value $=0.047)$ were positively linked. For the outcome as intimal thickening, age $(\mathrm{OR}=0.960,95 \% \mathrm{Cl}: 0.936$ to $0.984, \mathrm{P}$ value $=0.001)$, being female $(\mathrm{OR}=0.409,95 \% \mathrm{Cl}: 0.188$ to $0.889, \mathrm{p}$ value $=0.024)$ were negatively associated with the risk

Table 4

Result from multi-variable logistic regression

\begin{tabular}{|c|c|c|c|c|c|c|c|c|c|}
\hline \multirow[t]{2}{*}{ Potential Risk Factors } & \multicolumn{2}{|c|}{$\begin{array}{l}\text { Mean (SD)/n } \\
(\%)\end{array}$} & \multirow[t]{2}{*}{$\begin{array}{l}\mathrm{p} \\
\text { value }\end{array}$} & \multicolumn{2}{|c|}{$\begin{array}{l}\text { Mean (SD)/n } \\
(\%)\end{array}$} & \multirow[t]{2}{*}{$\begin{array}{l}\mathrm{p} \\
\text { value }\end{array}$} & \multicolumn{2}{|c|}{$\begin{array}{l}\text { Mean (SD)/n } \\
(\%)\end{array}$} & \multirow[t]{2}{*}{$\mathrm{p}_{\text {value }}$} \\
\hline & CD & Non-CD & & CA & $\begin{array}{l}\text { Non- } \\
\text { CA }\end{array}$ & & CIT & $\begin{array}{l}\text { Non- } \\
\text { CIT }\end{array}$ & \\
\hline Age (month) & 0.975 & $\begin{array}{l}(0.955) \\
0.994)\end{array}$ & $0.012^{*}$ & 0.962 & $\begin{array}{l}(0.933 \\
0.991)\end{array}$ & $0.012^{*}$ & 0.960 & $\begin{array}{l}(0.936 \\
0.984)\end{array}$ & $0.001 *$ \\
\hline $\begin{array}{l}\text { Gender (Female } \\
\text { compared to male) }\end{array}$ & 0.304 & $\begin{array}{l}(0.142, \\
0.648)\end{array}$ & $0.002^{*}$ & 0.483 & $\begin{array}{l}(0.195, \\
1.195)\end{array}$ & 0.116 & 0.409 & $\begin{array}{l}(0.188, \\
0.889)\end{array}$ & $0.024^{*}$ \\
\hline Duration of fever (day) & 0.962 & $\begin{array}{l}\text { (0.836) } \\
1.107)\end{array}$ & 0.586 & 1.128 & $\begin{array}{l}(0.975 \\
1.306)\end{array}$ & 0.106 & 1.015 & $\begin{array}{l}(0.893 \\
1.153)\end{array}$ & 0.818 \\
\hline $\begin{array}{l}\text { Time of initial use of } \\
\text { IVIG (day after the onsite } \\
\text { of KD) }\end{array}$ & 1.255 & $\begin{array}{l}(1.047 \\
1.503)\end{array}$ & $0.014 *$ & 1.214 & $\begin{array}{l}(1.003, \\
1.469)\end{array}$ & $0.047 *$ & 1.184 & $\begin{array}{l}(1.000 \\
1.401)\end{array}$ & 0.050 \\
\hline $\operatorname{ESR}(\mathrm{mm} / \mathrm{h})$ & 1.006 & $\begin{array}{l}(0.991 \\
1.021)\end{array}$ & 0.438 & 1.017 & $\begin{array}{l}(0.999 \\
1.036)\end{array}$ & 0.067 & 1.007 & $\begin{array}{l}\text { (0.991) } \\
1.022)\end{array}$ & 0.395 \\
\hline WBC count $\left(\times 10^{9} / \mathrm{L}\right)$ & 1.007 & $\begin{array}{l}(0.949 \\
1.067)\end{array}$ & 0.828 & 1.088 & $\begin{array}{l}(1.012, \\
1.169)\end{array}$ & $0.023 *$ & 1.030 & $\begin{array}{l}(0.969, \\
1.095)\end{array}$ & 0.336 \\
\hline $\begin{array}{l}\text { Time of PLT increase } \\
\text { (day after the onsite of } \\
\text { KD) }\end{array}$ & 0.999 & $\begin{array}{l}(0.998, \\
1.000)\end{array}$ & 0.210 & 1.000 & $\begin{array}{l}(0.999, \\
1.002)\end{array}$ & 0.753 & 0.999 & $\begin{array}{l}(0.998 \\
1.001)\end{array}$ & 0.341 \\
\hline $\begin{array}{l}\text { Maximum PLT count } \\
\left(\times 10^{9} / \mathrm{L}\right)\end{array}$ & 1.001 & $\begin{array}{l}(0.999 \\
1.003)\end{array}$ & 0.298 & 1.000 & $\begin{array}{l}(0.998, \\
1.002)\end{array}$ & 0.980 & 0.999 & $\begin{array}{l}(0.997 \\
1.001)\end{array}$ & 0.464 \\
\hline Albumin (g/L) & 0.926 & $\begin{array}{l}(0.858 \\
0.998)\end{array}$ & $0.045^{\star}$ & 0.890 & $\begin{array}{l}(0.809 \\
0.979)\end{array}$ & $0.017 *$ & 0.927 & $\begin{array}{l}(0.857 \\
1.002)\end{array}$ & 0.056 \\
\hline $\operatorname{lgG}(g / L)$ & 1.027 & $\begin{array}{l}(0.971 \\
1.086)\end{array}$ & 0.351 & 0.993 & $\begin{array}{l}(0.932 \\
1.059)\end{array}$ & 0.832 & 1.035 & $\begin{array}{l}(0.976 \\
1.097)\end{array}$ & 0.256 \\
\hline \multicolumn{10}{|l|}{${ }^{*} p<0.05$} \\
\hline
\end{tabular}

In the sensitivity analysis that added IVIG resistance as a covariance in each model (Table 5), the risk factors for coronary dilation and intimal thickening remained the same. Time of initial use of IVIG and WBC for coronary aneurysm became statistically insignificant. 
Table 5

Result from sensitivity analysis

\begin{tabular}{|c|c|c|c|c|c|c|c|c|c|}
\hline \multirow[t]{2}{*}{ Potential Risk Factors } & \multicolumn{2}{|c|}{$\begin{array}{l}\text { Mean (SD)/n } \\
(\%)\end{array}$} & \multirow[t]{2}{*}{$\begin{array}{l}p \\
\text { value }\end{array}$} & \multicolumn{2}{|c|}{$\begin{array}{l}\text { Mean (SD)/n } \\
(\%)\end{array}$} & \multirow[t]{2}{*}{$\begin{array}{l}p \\
\text { value }\end{array}$} & \multicolumn{2}{|c|}{$\begin{array}{l}\text { Mean (SD)/n } \\
(\%)\end{array}$} & \multirow[t]{2}{*}{$\begin{array}{l}p \\
\text { value }\end{array}$} \\
\hline & CD & Non-CD & & CA & $\begin{array}{l}\text { Non- } \\
\text { CA }\end{array}$ & & CIT & $\begin{array}{l}\text { Non- } \\
\text { CIT }\end{array}$ & \\
\hline IVIG resistance & 1.217 & $\begin{array}{l}(0.354 \\
4.188)\end{array}$ & 0.755 & 0.474 & $\begin{array}{l}(0.085 \\
2.637)\end{array}$ & 0.394 & 0.491 & $\begin{array}{l}(0.113 \\
2.134)\end{array}$ & 0.342 \\
\hline Age (month) & 0.975 & $\begin{array}{l}(0.956, \\
0.995)\end{array}$ & $0.014^{*}$ & 0.959 & $\begin{array}{l}(0.93 \\
0.990)\end{array}$ & $0.009 *$ & 0.958 & $\begin{array}{l}(0.934 \\
0.983)\end{array}$ & $0.001^{*}$ \\
\hline $\begin{array}{l}\text { Gender (Female } \\
\text { compared to male) }\end{array}$ & 0.304 & $\begin{array}{l}(0.143 \\
0.648)\end{array}$ & $0.002^{\star}$ & 0.469 & $\begin{array}{l}(0.188, \\
1.168)\end{array}$ & 0.104 & 0.402 & $\begin{array}{l}(0.184 \\
0.878)\end{array}$ & $0.022 *$ \\
\hline Duration of fever (day) & 0.952 & $\begin{array}{l}(0.815) \\
1.113)\end{array}$ & 0.538 & 1.163 & $\begin{array}{l}(0.988, \\
1.369)\end{array}$ & 0.070 & 1.047 & $\begin{array}{l}(0.910 \\
1.206)\end{array}$ & 0.518 \\
\hline $\begin{array}{l}\text { Time of initial use of } \\
\text { IVIG (day after the onsite } \\
\text { of KD) }\end{array}$ & 1.270 & $\begin{array}{l}(1.041 \\
1.550)\end{array}$ & $0.018 *$ & 1.172 & $\begin{array}{l}(0.953, \\
1.441)\end{array}$ & 0.133 & 1.140 & $\begin{array}{l}(0.949 \\
1.369)\end{array}$ & 0.160 \\
\hline $\mathrm{ESR}(\mathrm{mm} / \mathrm{h})$ & 1.006 & $\begin{array}{l}(0.991 \\
1.021)\end{array}$ & 0.456 & 1.018 & $\begin{array}{l}(1.000, \\
1.037)\end{array}$ & 0.053 & 1.008 & $\begin{array}{l}(0.992 \\
1.023)\end{array}$ & 0.339 \\
\hline WBC count $\left(\times 10^{9} / \mathrm{L}\right)$ & 1.006 & $\begin{array}{l}(0.949 \\
1.067)\end{array}$ & 0.844 & 1.094 & $\begin{array}{l}(1.016, \\
1.179)\end{array}$ & $0.017 *$ & 1.034 & $\begin{array}{l}(0.972 \\
1.100)\end{array}$ & 0.291 \\
\hline $\begin{array}{l}\text { Time of PLT increase } \\
\text { (day after the onsite of } \\
\text { KD) }\end{array}$ & 0.999 & $\begin{array}{l}(0.998 \\
1.001)\end{array}$ & 0.218 & 1.000 & $\begin{array}{l}(0.999 \\
1.002)\end{array}$ & 0.812 & 0.999 & $\begin{array}{l}(0.998 \\
1.001)\end{array}$ & 0.310 \\
\hline $\begin{array}{l}\text { Maximum PLT count } \\
\left(\times 10^{9} / \mathrm{L}\right)\end{array}$ & 1.001 & $\begin{array}{l}(0.999 \\
1.003)\end{array}$ & 0.298 & 1.000 & $\begin{array}{l}\text { (0.997, } \\
1.002)\end{array}$ & 0.952 & 0.999 & $\begin{array}{l}\text { (0.997, } \\
1.001)\end{array}$ & 0.463 \\
\hline Albumin $(\mathrm{g} / \mathrm{L})$ & 0.925 & $\begin{array}{l}(0.858 \\
0.998)\end{array}$ & $0.044^{\star}$ & 0.890 & $\begin{array}{l}(0.809, \\
0.980)\end{array}$ & 0.018 & 0.928 & $\begin{array}{l}(0.857 \\
1.003)\end{array}$ & 0.061 \\
\hline $\operatorname{lgG}(g / L)$ & 1.026 & $\begin{array}{l}(0.970 \\
1.085)\end{array}$ & 0.376 & 0.999 & $\begin{array}{l}(0.936, \\
1.065)\end{array}$ & 0.969 & 1.039 & $\begin{array}{l}(0.980 \\
1.102)\end{array}$ & 0.202 \\
\hline \multicolumn{10}{|l|}{${ }^{*} \mathrm{p}<0.05$} \\
\hline $\begin{array}{l}\text { CA: Coronary aneurysm; } \\
\text { rate; IVIG: intravenous im } \\
\text { white blood cell }\end{array}$ & $\begin{array}{l}\text { Corona } \\
\text { loglob }\end{array}$ & $\begin{array}{l}\text { dilation; } \\
\text {; LDH: I }\end{array}$ & $\begin{array}{l}\text { T: Coro } \\
\text { ic dehy }\end{array}$ & y intin & $\begin{array}{l}\text { thicke } \\
\text { PLT: pl }\end{array}$ & g; ESR: & $\begin{array}{l}\text { throcy } \\
\text { andarc }\end{array}$ & $\begin{array}{l}\text { sedime } \\
\text { eviation }\end{array}$ & $\begin{array}{l}\text { tion } \\
\text { 'BC: }\end{array}$ \\
\hline
\end{tabular}

\section{Discussion}

In total, 260 patients with infantile KD were recruited in our study, with a mean age of 24 month. Sixty-six (25.4\%), thirty-nine (15\%) and fifty-six patients (21.5\%) of the participants had coronary dilation, coronary aneurysm, and intimal thickening of coronary arteries, respectively. Single-variable analysis found age, gender, duration of fever, time of initial use of IVIG, ESR, WBC, time of platelet increase, maximum value of platelet, albumin and lgG level were associated with CAL. In multi-variable logistic analysis, younger in age, male, lower serum albumin level, and later initial use of IVIG were found to be potential risk factors of CAL in the studied population. In the sensitivity analysis that added IVIG resistance as a covariance in the models, the risk factors for coronary dilation and intimal thickening remained the same. 
There are few studies that explored the association between biomarkers and CAL in KD patients. A number of them found similar results to our study, suggested that lower albumin was a risk factor of CAL in KD patients [20,21]. Meanwhile, other risk factors have also been revealed in the current literature, such as hemoglobin, fever duration, Creactive protein, and so on. For instance, H.J. Kim et al reported that compared to KD patients without CAL, those with CAL had significantly higher serum interleukin-6 levels $(228.26 \mathrm{ng} / \mathrm{mL}$ vs. $39.18 \mathrm{ng} / \mathrm{mL})$, and lower body mass index (15.09 vs. 16.60) [22]. Li et al. revealed that red blood cell distribution width could be used as an independent predictor of CAL in KD patients [23]. Shao et al. found Apo-A level, as a predictor of CAL in KD patients, was significantly negatively linked to CAL [24]. In our study, factors such as duration of fever and platelet level were not found to be significantly associated with CAL. The variance in ethnicity, geographic location and seasonality could partially explain the divergences in our findings. In addition, a large body of evidence involved research on potential predictive biomarkers of CAL in KD patients, which were novelty but could not be easily measured and monitored in the general medical practice. The biomarkers included adropin [20], plasminogen activator inhibitor-1 [25], tenascin-C [26], nterminal pro-brain natriuretic peptide [27-33], circulating endothelial glycocalyx components [26], lymphocyte hydrogen sulfide production [28], to mention a few. Different from these studies, we included results from clinical blood examinations as potential risk factors, which could be easier and faster to be assessed in the clinical practice. Furthermore, extracoronary echocardiographic findings and Z-values were also found to have predictive value for CAL $[28,33,34]$. Compared to those studies, investigation on blood-based biomarkers avoided the influence of variance in equipment and technicians.

In our study, lower albumin level was found to be a risk factor of CAL. Serum albumin is commonly used as a parameter to negatively indicate the activity of inflammatory as an acute phase reactant [35]. Hypoalbuminemia in KD patients was reported to be caused by vascular leakage, which was due to the elevation of vascular endothelial growth factor. Studies have found that vascular endothelial growth factor and its receptors (Fms-related tyrosine kinase- 1 and kinase insert domain receptor) were involved in the development and progression of CAL in KD patients [36-38].

Although IVIG combined with aspirin were commonly adopted as the mainstay of initial treatment which could effectively decrease the development of CAL in KD patients, the high incidence of IVIG-resistant were reported in recent years [39-43]. Approximately 10-20\% of pediatric patients with KD were reported to have persistent or recrudescent fever after the initial IVIG therapy $[44,45]$. Patients that were not responsive to IVIG therapy were considered as at higher risk of incidence for CAL $[40,43]$. Several studies suggested potential underlying mechanism that could partially explain the phenomenon. For example, through genome-wide association studies (GWAS), a number of genetic markers associated with both IVIG resistance and CAL formation were found in KD patients, including inositol 1,4,5-trisphosphate 3-kinase C (ITPKC), caspase-3 (CASP3), FCGR2A, CD40, and interleukin1 beta (IL-1B) [46, 47]. Wang et al. found that the change of serum levels of IL-10 varied between patients with and without IVIG resistance after the usage of the medication, and the genetic polymorphisms of IL-10 were corelated to CAL [48]. However, in our study, IVIG resistance was not found to be associated with CAL. The potential reasons could be as followed. The IVIG resistance may suggest the severity of ongoing inflammation, nevertheless development of CAL might also be affected by other factors such as hemodynamics [49], of which the data were not collected in our study. Moreover, in the sensitivity analysis that involved IVIG resistance, even though the responsiveness of IVIG was not found to be associated with CAL directly, some of the other risk factors such as WBC and time of initial use of IVIG became nonsignificant after the addition of IVIG resistance. The mechanisms that lead to the variation should be investigated in future research.

It is crucial to identify the patients with elevated risk of CAL at early stage of the disease, through surveillance on specific risk factors in KD patients. Our study suggested several risk factors including age, gender and albumin, with 
might be useful in clinical practice because these factors are easy to be measured and are possible to be tested frequently. It could help the pediatrician identify patients that might at high risk of CAL at early stage of the disease, for example upon admission to the hospital. Meanwhile, our findings could offer the pediatrician guidance on monitoring and providing targeted preventive and therapeutic measures. It could be inferred that patients with KD could be benefited from the timely medical treatment.

\section{Strength and limitations}

Our present study provided evidence on the associations between clinical biomarkers and the incidence of several CALs. There are a plenty of strengths that made our study valuable and practical. First, although the study was retrospective, we used data from medical records, which were obtained during the hospitalization. The accuracy and reliability of the data largely reduced the potential recall bias. Second, by including a group of clinical biomarkers as potential risk factors, combined with a comprehensive involvement of three major outcomes, our study provided an overall insight of the topic. Third, KD has been more prevalent in Asia area. Our study was carried out in Asian population (Chinese), which made our result proper to be generalized in the high-risk area of Asia. Forth, we investigated clinical characteristics and biomarkers that are easy to be examined, which made our conclusion more practical to be verified in future clinical research, and hence, to be implemented in clinics. However, there are several limitations of our study. First, the duration of follow-up was short. KD could have a life-long influence on the patients' health conditions. As reported by a Canadian study, $5 \%$ miocardial infarction patients less than 40 years old who underwent coronary angiography had lesions that were linked to sequelae of KD [50]. Therefore, it is necessary to follow up the participants as long as possible, to investigate the longitudinal risk factors for CAL. Second, the sample size of our study was relevant small. Due to the low incidence of KD in Guangdong province (approximately 5 per 100000) [7], it might be greatly time-consuming to recruit a large sample, even in multi-center research. Because of the insufficient sample, we cannot compare our findings between IVIG responsive and nonresponsive patients. The small sample size weakened the robustness of our conclusion. Third, information on some of the potential confounders that could have influence on the result were not collected in our study. The factors included family history, diet, sleep and other life-style factors, genetic data, etc. These data could not be implemented due to the retrospective feature of the study. Forth, we did not include patients in the recurrent episode. However, Nakamura et al. reported a higher risk of developing coronary artery sequelae with the recurrent episode [12]. This characteristic of our sample limited the generalization of our conclusion.

\section{Conclusion}

Our study analyzed data from a Chinese pediatric population with KD, and found that younger in age, male, lower serum albumin lever, and later initial use of IVIG were risk factors of CAL in this population. Our findings had high practicability to be implemented in clinical practice, however, future prospective multi-center studies, with longer follow-up duration, and involving more comprehensive exposure factors are essential to further verify our conclusion.

\section{Declarations}

\section{Ethics approval and consent to participate}

The protocol of the present study was reviewed and approved by the Guangzhou Women and Children Medical Center Ethics Committee (2021241A01) and was conducted according to the International Ethical Guidelines for Research Involving Human Subjects stated in the Declaration of Helsinki. The children's families provide written informed consent. 


\section{Consent for publication}

Our manuscript does not contain information or images that could lead to identification of a study participant.

\section{Availability of data and materials}

The datasets generated and analysed during the current study are not publicly available due to the requirements of the hospital, but are available from the corresponding author on reasonable request.

\section{Competing interests}

The authors declare no competing interests.

\section{Funding}

This work was supported by Research project of Traditional Chinese Medicine Bureau of Guangdong Province (Grant no. :20201269, Grant recipient: Xiaogang Chen; Grant no. :20212175, Grant recipient: Xiangna Yang)

\section{Authors' contribution}

Conception and design: $\mathrm{XY}, \mathrm{XC}$; Data collection: $\mathrm{XY}, \mathrm{JD}, \mathrm{YW}$; Analysis and interpretation of data: $\mathrm{XY}, \mathrm{ZL}$; Writing the

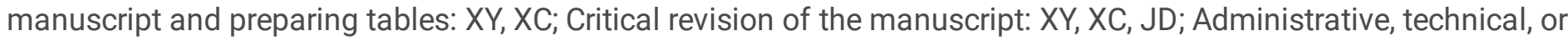
material support: $X Y, X C, Y W$; Obtaining funding: $X Y, X C$; Supervision: $X C$; Review of the manuscript: all authors.

\section{Acknowledgements}

The authors acknowledge and thank all of the participating patients and their families.

\section{References}

1. Saundankar, J., et al., The epidemiology and clinical features of Kawasaki disease in Australia. Pediatrics, 2014. 133(4): p. e1009-14.

2. Maddox RA, et al., Monitoring the occurrence of Kawasaki syndrome in the United States, in Proceedings of the Eleventh International Kawasaki Disease Symposium. 2015: Hawaii, United States.

3. Uehara, R. and E.D. Belay, Epidemiology of Kawasaki disease in Asia, Europe, and the United States. J Epidemiol, 2012. 22(2): p. 79-85.

4. Makino, N., et al., Descriptive epidemiology of Kawasaki disease in Japan, 2011-2012: from the results of the 22nd nationwide survey. J Epidemiol, 2015. 25(3): p. 239-45.

5. Huang, W.C., et al., Epidemiologic features of Kawasaki disease in Taiwan, 2003-2006. Pediatrics, 2009. 123(3): p. e401-5.

6. Chang, L.Y., et al., Epidemiologic features of Kawasaki disease in Taiwan, 1996-2002. Pediatrics, 2004. 114(6): p. e678-82.

7. Xiaoguang Zhou, Shuzhen Wang, and e. al., Epidemiology of Kawasaki disease in children in Guangdong. Chinese Journal of Epidemiology, 2003(01). 
8. Du, Z.D., et al., Epidemiologic picture of Kawasaki disease in Beijing from 1995 through 1999. Pediatr Infect Dis J, 2002. 21(2): p. 103-7.

9. Singh, S., P. Vignesh, and D. Burgner, The epidemiology of Kawasaki disease: a global update. Arch Dis Child, 2015. 100(11): p. 1084-8.

10. Singh, S., et al., Is Kawasaki disease incidence rising in Chandigarh, North India? Arch Dis Child, 2011. 96(2): p. 137-40.

11. Holman, R.C., et al., Racial/ethnic differences in the incidence of Kawasaki syndrome among children in Hawaii. Hawaii Med J, 2010. 69(8): p. 194-7.

12. Tissandier, C., et al., Kawasaki shock syndrome complicating a recurrence of Kawasaki disease. Pediatrics, 2014. 134(6): p. e1695-9.

13. Fujiwara, H. and Y. Hamashima, Pathology of the heart in Kawasaki disease. Pediatrics, 1978. 61(1): p. $100-7$.

14. Nakamura, Y., et al., Mortality among persons with a history of kawasaki disease in Japan: mortality among males with cardiac sequelae is significantly higher than that of the general population. Circ J, 2008. 72(1): p. 134-8.

15. Nakamura, Y., et al., Mortality among Japanese with a history of Kawasaki disease: results at the end of 2009. J Epidemiol, 2013. 23(6): p. 429-34.

16. Burns, J.C., et al., Sequelae of Kawasaki disease in adolescents and young adults. J Am Coll Cardiol, 1996. 28(1): p. 253-7.

17. Rife, E. and A. Gedalia, Kawasaki Disease: an Update. Curr Rheumatol Rep, 2020. 22(10): p. 75.

18. Newburger, J.W., et al., Diagnosis, treatment, and long-term management of Kawasaki disease: a statement for health professionals from the Committee on Rheumatic Fever, Endocarditis and Kawasaki Disease, Council on Cardiovascular Disease in the Young, American Heart Association. Circulation, 2004. 110(17): p. $2747-71$.

19. McCrindle, B.W., et al., Diagnosis, Treatment, and Long-Term Management of Kawasaki Disease: A Scientific Statement for Health Professionals From the American Heart Association. Circulation, 2017. 135(17): p. e927e999.

20. Yang, M., et al., Association between adropin and coronary artery lesions in children with Kawasaki disease. Eur J Pediatr, 2021. 180(7): p. 2253-2259.

21. Kim, H.J., E.H. Choi, and H.R. Kil, Association between adipokines and coronary artery lesions in children with Kawasaki Disease. J Korean Med Sci, 2014. 29(10): p. 1385-90.

22. Shao, S., et al., Predictive Value of Serum Lipid for Intravenous Immunoglobulin Resistance and Coronary Artery Lesion in Kawasaki Disease. J Clin Endocrinol Metab, 2021. 106(10): p. e4210-e4220.

23. Ming, L., et al., Red Blood Cell Distribution Width as a Predictive Marker for Coronary Artery Lesions in Patients with Kawasaki Disease. Pediatr Cardiol, 2021. 42(7): p. 1496-1503.

24. Okuma, Y., et al., Serum Tenascin-C as a Novel Predictor for Risk of Coronary Artery Lesion and Resistance to Intravenous Immunoglobulin in Kawasaki Diseaseã 奴- A Multicenter Retrospective Study. Circ J, 2016. 80(11): p. 2376-2381.

25. Senzaki, H., et al., Plasminogen activator inhibitor-1 in patients with Kawasaki disease: diagnostic value for the prediction of coronary artery lesion and implication for a new mode of therapy. Pediatr Res, 2003. 53(6): p. 9838.

26. Ohnishi, Y., et al., Circulating endothelial glycocalyx components as a predictive marker of coronary artery lesions in Kawasaki disease. Int J Cardiol, 2019. 292: p. 236-240. 
27. Huiling, L., L. Yaping, and H. Xiufen, [Prediction of the risk of coronary arterial lesions in Kawasaki disease by Nterminal pro-brain natriuretic peptide]. Zhonghua Er Ke Za Zhi, 2015. 53(4): p. 300-3.

28. Bilal, M., et al., The Importance of Serum N-Terminal Pro-Brain Natriuretic Peptide and Endogenous Hydrogen Sulfide for Predicting Coronary Artery Lesions in Pediatric Kawasaki Disease Patients: Findings From a Tertiary Care Hospital in Karachi, Pakistan. Cureus, 2020. 12(7): p. e9016.

29. Chen, Y.L., J.L. Wang, and W.Q. Li, Prediction of the risk of coronary arterial lesions in Kawasaki disease by serum 25-hydroxyvitamin D3. Eur J Pediatr, 2014. 173(11): p. 1467-71.

30. Chang, L.S., et al., Neutrophil-to-lymphocyte ratio and scoring system for predicting coronary artery lesions of Kawasaki disease. BMC Pediatr, 2020. 20(1): p. 398.

31. Hua, W., et al., A new scoring system to predict Kawasaki disease with coronary artery lesions. Clin Rheumatol, 2019. 38(4): p. 1099-1107.

32. Cho, H.J., et al., The Risk Prediction of Coronary Artery Lesions through the Novel Hematological Z-Values in 4 Chronological Age Subgroups of Kawasaki Disease. Medicina (Kaunas), 2020. 56(9).

33. Lega, J.C., et al., Extracoronary echocardiographic findings as predictors of coronary artery lesions in the initial phase of Kawasaki disease. Arch Dis Child, 2013. 98(2): p. 97-102.

34. Sun, Y., et al., Plasma H2S predicts coronary artery lesions in children with Kawasaki disease. Pediatr Int, 2015. 57(5): p. 840-4.

35. Al-Subaie, N., et al., C-reactive protein as a predictor of outcome after discharge from the intensive care: a prospective observational study. Br J Anaesth, 2010. 105(3): p. 318-25.

36. Terai, M., et al., Prognostic impact of vascular leakage in acute Kawasaki disease. Circulation, 2003. 108(3): p. 325-30.

37. Maeno, N., et al., Increased serum levels of vascular endothelial growth factor in Kawasaki disease. Pediatr Res, 1998. 44(4): p. 596-9.

38. Kariyazono, H., et al., Association of vascular endothelial growth factor (VEGF) and VEGF receptor gene polymorphisms with coronary artery lesions of Kawasaki disease. Pediatr Res, 2004. 56(6): p. 953-9.

39. Mori, M., et al., Predictors of coronary artery lesions after intravenous gamma-globulin treatment in Kawasaki disease. J Pediatr, 2000. 137(2): p. 177-80.

40. Ashouri, N., et al., Risk factors for nonresponse to therapy in Kawasaki disease. J Pediatr, 2008. 153(3): p. 365-8.

41. Tremoulet, A.H., et al., Resistance to intravenous immunoglobulin in children with Kawasaki disease. J Pediatr, 2008. 153(1): p. 117-21.

42. Ruan, Y., B. Ye, and X. Zhao, Clinical characteristics of Kawasaki syndrome and the risk factors for coronary artery lesions in China. Pediatr Infect Dis J, 2013. 32(10): p. e397-402.

43. Guidelines for diagnosis and management of cardiovascular sequelae in Kawasaki disease (JCS 2013). Digest version. Circ J, 2014. 78(10): p. 2521-62.

44. Egami, K., et al., Prediction of resistance to intravenous immunoglobulin treatment in patients with Kawasaki disease. J Pediatr, 2006. 149(2): p. 237-40.

45. Durongpisitkul, K., et al., Immunoglobulin failure and retreatment in Kawasaki disease. Pediatr Cardiol, 2003. 24(2): p. 145-8.

46. Onouchi, Y., et al., ITPKC and CASP3 polymorphisms and risks for IVIG unresponsiveness and coronary artery lesion formation in Kawasaki disease. Pharmacogenomics J, 2013. 13(1): p. 52-9. 
47. Lee, Y.C., et al., Two new susceptibility loci for Kawasaki disease identified through genome-wide association analysis. Nat Genet, 2012. 44(5): p. 522-5.

48. Wang, Y., et al., Evaluation of intravenous immunoglobulin resistance and coronary artery lesions in relation to Th1/Th2 cytokine profiles in patients with Kawasaki disease. Arthritis Rheum, 2013. 65(3): p. 805-14.

49. Kobayashi, T., et al., Prediction of intravenous immunoglobulin unresponsiveness in patients with Kawasaki disease. Circulation, 2006. 113(22): p. 2606-12.

50. Daniels, L.B., et al., Prevalence of Kawasaki disease in young adults with suspected myocardial ischemia. Circulation, 2012. 125(20): p. 2447-53. 